\title{
Past 24 to 48 Hours
}

National Cancer Institute

\section{Source}

National Cancer Institute. Past 24 to 48 Hours. NCI Thesaurus. Code C131915.

The 24-48 hour period occurring just before the present time. 